\title{
Evaluación del componente infantil del Seguro Público de Salud de la Provincia de Buenos Aires
}

\author{
Natalia Yavich, D en CS, ${ }^{(1,2)}$ Ernesto Pablo Báscolo, D en CS,(1) Jeannie Haggerty, PhD en E. (2)
}

\author{
Yavich N, Báscolo EP, Haggerty J. \\ Evaluación del componente infantil del Seguro \\ Público de salud de la Provincia de Buenos Aires. \\ Salud Publica Mex 2013;55:26-34.
}

\section{Resumen}

Objetivo. Evaluar si el Seguro Público de Salud de la Provincia de Buenos Aires fortaleció el papel del primer nivel de atención y mejoró el acceso y la integralidad de la atención. Material y métodos. En 2006 se realizó una encuesta domiciliaria en ocho municipios $(n=24 / 3)$. Se recolectó información sobre utilización de servicios entre beneficiarios del Seguro Público de Salud menores de ocho años. Se aplicaron pruebas de hipótesis para captar diferencias significativas entre beneficiarios con distintos patrones de uso de servicios y perfiles municipales de implementación del Seguro. Resultados. Más de $95 \%$ de los beneficiarios recibieron atención utilizando el Seguro u otros servicios. El uso del Seguro varió significativamente entre perfiles municipales. Los beneficiarios que utilizaron el Seguro y otros servicios lograron una mayor integralidad de la atención y acceso a consultas médicas que quienes utilizaron el Seguro exclusivamente. El uso de hospitales fue significativamente menor entre usuarios del Seguro. Conclusiones. El Seguro fue más efectivo al estar articulado con la red de servicios municipales.

Palabras clave: accesibilidad a los servicios de salud; atención primaria de salud; evaluación de servicios de salud; atención integral de salud; integración de sistemas
Yavich N, Báscolo EP, Haggerty J.

Evaluation of the child component of the

Buenos Aires Public Health Insurance.

Salud Publica Mex 2013;55:26-34.

\section{Abstract}

Objective. Evaluate if the Insurance strengthened the primary care role and enhanced healthcare access and comprehensiveness. Materials and methods. Through a household survey $(n=24 \mid 3$; year 2006) we collected data on utilization of health services by under 8 year old beneficiaries from 8 municipalities. We used hypothesis tests to identify significant differences between municipal profiles of Insurance implementation and beneficiaries grouped by utilization patterns. Results. Although more than $95 \%$ of children received medical healthcare in each municipality (using the Insurance and other services), the Insurance utilization varied significantly by municipal profile. Access to comprehensive healthcare and consultation rates were significantly higher for those beneficiaries who used the Insurance along with other health services, compared with those who only used the Insurance services. Use of hospitals was significantly lower within Insurance users. Conclusion. The Insurance was more effective when implemented and used in conjunction with the municipal networks of health services.

Key words: health services accessibility; primary health care; health services evaluation; comprehensive health care; systems integration

(I) Maestría en Gestón de Sistemas y Servicios de Salud, Centro de Estudios Interdisciplinarios, Universidad Nacional de Rosario. Argentina.

(2) McGill University, Department of Family Medicine, St. Mary's Hospital Research Centre. Montreal, Canada.

Fecha de recibido: 17 de abril de 2012 - Fecha de aceptado: 16 de octubre de 2012 Autor de correspondencia: Natalia Yavich. Alsina 1069. Rosario (2000), Provincia de Santa Fe, Argentina Correo electrónico: nyavich@gmail.com 
$\mathrm{E}^{\mathrm{n}}$ el marco de las políticas de descentralización, numerosos países latinoamericanos han implementado programas locales y nacionales orientados a mejorar el acceso y la calidad de la atención, o a reformar el modelo de atención de las redes locales de servicios de salud. ${ }^{1-5}$ Una estrategia ampliamente utilizada ha sido la contratación de profesionales o equipos de salud con el fin de promover un nuevo modelo de atención y gestión. La articulación de los programas centrales con las redes de servicios locales es un factor clave para evitar la fragmentación y promover la integralidad de la atención, dado que comparten una misma población objetivo y necesitan complementarse para brindar una atención integral. 6,7

El Ministerio de Salud creó el Seguro Público de Salud de la Provincia de Buenos Aires, con el fin de fortalecer su rectoría sobre los municipios y promover la reforma del primer nivel municipal. Sus objetivos fueron fortalecer la estrategia de atención primaria de la salud y mejorar la continuidad, acceso e integralidad de los servicios de salud. ${ }^{8}$ En este trabajo se busca evaluar si el componente infantil del Seguro alcanzó tales objetivos.

Según Peter Evans, el éxito del Estado en la implementación de los cambios está determinado por las alianzas que el organismo promotor del cambio logra construir con otros actores del Estado y de la sociedad civil. ${ }^{9}$ En el caso del Seguro, las alianzas con las secretarías de salud municipales estaban atravesadas por tensiones dadas entre el interés del Seguro por reformar el modelo de gestión y atención del primer nivel municipal, y la intención de las secretarias de mantener su autonomía en el manejo del primer nivel de atención. 10,11

Entre octubre de 2004 y diciembre de 2006, se llevó a cabo la investigación "Análisis de las capacidades institucionales y evaluación del desempeño del Seguro Público de Salud de la Provincia de Buenos Aires (SPS)," con el apoyo del International Development Research Centre de Canadá (IDRC) y la Organización Panamericana de la Salud (OPS). En el marco de dicho estudio, se caracterizaron ocho municipios de la Provincia de Buenos Aires en función de su perfil de implementación del Seguro. En este trabajo se evalúa el desempeño del Seguro, considerando los distintos perfiles municipales de implementación, mismos que reflejan la naturaleza de las alianzas y la modalidad de integración entre los servicios municipales y los del Seguro. ${ }^{10}$ A continuación se describe cada uno:

1. Implementación integrada: la Secretaría de Salud Municipal integra los recursos del Seguro con la red municipal para fortalecer su primer nivel de atención.
2. Integración "complementaria": la Secretaría de Salud utiliza el Seguro para la resolución de consultas preventivas y programadas, y a la red municipal para la resolución de urgencias y emergencias.

3. Implementación en paralelo: ambas redes funcionan en paralelo. Cuando los servicios municipales identifican a un paciente como beneficiario del Seguro, derivan su atención.

4. Implementación competitiva: el Seguro y la Secretaría de Salud "compiten" por la captación y fidelización de pacientes y no coordinan acciones.

5. Rechazo a la implementación: la Secretaría de Salud rechaza y obstaculiza la implementación del Seguro.

El lector interesado en conocer mayores detalles sobre el desarrollo de esta clasificación, puede consultar la bibliografía referida. ${ }^{10}$ En el cuadro I se ofrece información sobre las características de los municipios integrados en cada categoría.

Enseguida se indican los interrogantes abordados en este trabajo:

1. El uso del Seguro ¿está asociado con el nivel de acceso a los servicios de salud? Las diferencias de acceso a otros servicios y de uso del Seguro ¿son significativas entre perfiles municipales de implementación del Seguro?

2. ¿Contribuye el Seguro a mejorar la integralidad de la atención recibida por sus beneficiarios? ¿Existen diferencias significativas en la obtención de atención preventiva y curativa entre perfiles municipales de implementación del Seguro?

3. ¿Logra el Seguro fortalecer el papel del primer nivel de atención como puerta de entrada y fuente habitual de atención? Los usuarios del Seguro ¿utilizan menos los hospitales que los no usuarios del Seguro? ¿Son estas diferencias significativas entre perfiles municipales de implementación del Seguro?

\section{Descripción del Seguro}

El Seguro es un programa, creado a inicios de la década del 2000, destinado a brindar cobertura explícita a un conjunto nominal de beneficiarios. Esta modalidad de aseguramiento lo diferencia de otros programas y servicios del sistema de salud argentino, que asumen responsabilidad geográfica sobre la población bajo un modelo de financiamiento a la oferta.

Para poner en marcha el Seguro, se generaban acuerdos con las secretarías de salud municipales, en colaboración con otros actores locales como las secretarías de acción social, organizaciones no gubernamentales, 


\section{Cuadro I}

Municipios* agrupados por perfiles municipales de implementación del Seguro. Provincia de Buenos Aires, 2006 Proyecto I. "Análisis de las Capacidades institucionales y eValuación del desempeño del SPS"

\begin{tabular}{|c|c|c|c|c|c|c|}
\hline $\begin{array}{l}\text { Perfiles municipales de implementación y } \\
\text { número de municipios por clase }\end{array}$ & Tipo de municipio & Población municipal & $\begin{array}{c}\text { Número } \\
\text { de encuestados }\end{array}$ & $\begin{array}{l}\text { \% niños menores } \\
\text { de dos años }\end{array}$ & $\begin{array}{l}\% \text { respondientes } \\
\text { madres }\end{array}$ & $\begin{array}{c}\% \text { respondientes cuya escolaridad es }= \\
0<\text { a siete años de estudio }\end{array}$ \\
\hline Implementación integrada (2) & - Gran Buenos Aires & $\begin{array}{l}276190 \\
459263\end{array}$ & 603 & 18.6 & 95 & 79.6 \\
\hline Integración "complementaria” (I) & $\begin{array}{l}\text { - Semirrural } \\
\text { - Gran Buenos Aires }\end{array}$ & $\begin{array}{l}104590 \\
552902\end{array}$ & 604 & 18.7 & 93.9 & 81.1 \\
\hline Implementación en paralelo (I) & $\begin{array}{l}\text { - Mancha urbana alrededor } \\
\text { del Gran Buenos Aires }\end{array}$ & 299077 & 302 & 13.6 & 99 & 87 \\
\hline Implementación competitiva (2) & - Gran Buenos Aires & $\begin{array}{l}616279 \\
426005\end{array}$ & 605 & 21.2 & 90.5 & 79.5 \\
\hline Rechazo a la implementación (I) & - Gran Buenos Aires & 1775816 & 299 & 24.1 & 92.3 & 78.5 \\
\hline Total / Promedio & & & 2413 & 19.2 & 93.8 & 80.8 \\
\hline
\end{tabular}

* Municipios: Almirante Brown, Florencio Varela, La Matanza, Lanús, Lomas de Zamora, Pergamino, Pilar, San Miguel

Fuente: Referencias 10, 12

asociaciones profesionales, etcétera. En los casos en que las secretarías de salud no estaban interesadas en la implementación del Seguro, el proceso se llevaba a cabo sin su liderazgo o participación. En el año 2006, el Seguro contaba con más de 600000 beneficiarios de 46 municipios. $^{13}$

La población objetivo del Seguro estaba conformada por beneficiarios y trabajadores barriales de un programa provincial de ayuda alimentaria para familias socioeconómicamente vulnerables, integradas por mujeres, puérperas o niños desde los seis meses de edad hasta el ingreso a la escuela. La cobertura incluía servicios médicos ambulatorios y odontológicos; acceso gratuito a servicios de laboratorio (a través de laboratorios privados); medicamentos esenciales (provistos por el Programa Nacional REMEDIAR), y servicios de mediana y alta complejidad provistos a través de la red pública de hospitales y programas provinciales. ${ }^{8}$ Los beneficiarios recibían atención médica a través de un médico generalista, o un médico familiar o pediatra, cuyo consultorio debía estar ubicado en las inmediaciones del lugar de residencia de su población. Cada municipio podía ubicar sus médicos en centros de salud municipales o en organizaciones comunitarias (p. ej. clubes, comedores, sociedades de fomento, iglesias, etc.). Mediante la asignación de una cápita nominal de beneficiarios a cada médico y la introducción de incentivos económicos a los profesionales a fin de promover el contacto de estos últimos con sus pacientes, se buscaba fortalecer el acceso, continuidad e integralidad de la atención y el primer nivel de atención como puerta de entrada.

El hecho de ser beneficiario del Seguro no constituía un impedimento formal para el uso de los servicios de salud municipales. Si bien se esperaba que la población optara por hacer uso del Seguro en vista de las ventajas ofrecidas, la población podía acudir a los servicios municipales.

\section{Material y métodos}

En 2006 se aplicó una encuesta domiciliaria en ocho municipios de la Provincia de Buenos Aires. Para seleccionarlos, previamente se llevó a cabo un análisis factorial utilizando indicadores socioeconómicos y de salud de la población municipal, a través del cual se obtuvieron cuatro clusters. A partir de un muestreo intencional orientado a lograr la máxima heterogeneidad en cuanto a la capacidad municipal de gestión del Seguro, se seleccionaron dos municipios por cluster.

Se determinó un tamaño muestral de 300 beneficiarios por municipio. Se seleccionaron 2400 hogares, mediante un proceso estratificado y multietápico, controlado por la ubicación de los consultorios -respecto al lugar de residencia de los beneficiarios y a su cercanía con las zonas céntricas- y el lugar de instalación de los consultorios, dentro de una organización comunitaria o dentro de un servicio de salud municipal.

El objetivo de las entrevistas era conocer la utilización que se hacía de los servicios de salud, las barreras de acceso y la capacidad de respuesta del Seguro durante los 12 meses previos a la entrevista, así como las características demográficas y socioeconómicas de los hogares de beneficiarios menores de ocho años de edad. Los adultos a cargo de los beneficiarios fueron quienes proporcionaron las entrevistas. En los hogares con más de un beneficiario, se seleccionó uno en forma 
aleatoria. El cuestionario tuvo una duración promedio de 15 minutos.

La encuesta se complementó con entrevistas individuales y grupos focales en los ocho municipios, en los que participaron 163 madres de beneficiarios del Seguro y del Plan Vida (99 en 2005 y 64 en 2006). A través de los mismos, se profundizó en la comprensión de los resultados de la encuesta. Se indagaron los siguientes ejes: patrones de utilización de servicios, barreras de acceso y capacidad de respuesta del Seguro. Las entrevistas fueron transcritas y analizadas utilizando la técnica de análisis de contenido.

Para responder las interrogantes, se distinguieron tres patrones de utilización de servicios:

a) Seguro exclusivo: beneficiarios que durante el último año utilizaron sólo los servicios del Seguro.

b) Seguro y otros: beneficiarios que durante el último año utilizaron los servicios del Seguro y otras fuentes de atención, principalmente centros de salud municipales y hospitales públicos.

c) Otros servicios: beneficiarios que durante el último año obtuvieron atención sin utilizar el Seguro, utilizando principalmente centros de salud municipales y hospitales públicos.

Se aplicaron pruebas de hipótesis para captar diferencias significativas entre perfiles municipales de implementación del Seguro y patrones de utilización. Se utilizó la versión 19 del programa SPSS para llevar a cabo el análisis estadístico.

A continuación, se describen los métodos que se emplearon para dar respuesta a las tres interrogantes.

1. El uso del Seguro ¿está asociado con el nivel de acceso a los servicios de salud? Las diferencias de acceso a otros servicios y de uso del Seguro ison significativas entre perfiles municipales de implementación del Seguro?

El acceso se midió a través de la obtención de atención médica. ${ }^{14}$ La presencia de mejoras en el acceso se probó mediante el cálculo de la ji cuadrada de Pearson, para identificar las diferencias significativas entre perfiles municipales de implementación del Seguro, respecto de los porcentajes de beneficiarios que recibieron atención médica utilizando y sin utilizar el Seguro.

2. ¿Contribuye el Seguro a mejorar la integralidad de la atención recibida por sus beneficiarios? ¿Existen diferencias significativas en la obtención de atención preventiva y curativa entre perfiles municipales de implementación del Seguro?
El término integralidad hace referencia a la capacidad de satisfacer las necesidades de atención preventiva, curativa y de rehabilitación, a través de uno o más servicios. ${ }^{15}$ La integralidad fue medida como la obtención de atención preventiva y curativa durante el último año. La categoría de consultas preventivas refiere a consultas de control de niño sano. Las consultas agrupadas bajo la categoría de consultas curativas corresponden a consultas por problemas de salud nuevos o preexistentes con fines de diagnóstico, tratamiento o control.

Se calculó la ji cuadrada de Pearson para captar diferencias significativas en la obtención de atención exclusivamente curativa, exclusivamente preventiva o ambas, entre beneficiarios agrupados por patrones de uso de servicios y perfiles municipales de implementación del Seguro.

Se llevó a cabo el test de ANOVA para captar diferencias significativas respecto de las tasas de consultas preventivas y curativas entre beneficiarios agrupados por patrones de utilización de servicios y perfiles municipales de implementación del Seguro.

3. ¿Logra el Seguro fortalecer el rol del primer nivel de atención como puerta de entrada y fuente habitual de atención? Los usuarios del Seguro ¿utilizan menos los hospitales que los no usuarios del Seguro? ¿Son estas diferencias significativas entre perfiles municipales de implementación del Seguro?

A partir de la frecuencia del uso de hospitales, se infirió el fortalecimiento del primer nivel de atención como puerta de entrada. Este abordaje se basa en dos premisas: pediatras y generalistas están capacitados para resolver más de $80 \%$ de los motivos de consulta. ${ }^{13,16,17}$ Por lo tanto, la población que requiere atención médica especializada debería ser limitada y equivalente en cada municipio, dado que la edad y las condiciones socio-económicas de la población son similares en todos los municipios. La sobreutilización del hospital -y principalmente su uso para consultas preventivas- se explica por las barreras de acceso y por una baja capacidad de respuesta del primer nivel de atención. Esta segunda premisa surge de los hallazgos de un estudio realizado con la población objetivo del Seguro, donde se probó la asociación entre un mayor uso del segundo nivel de atención y una mayor debilidad del primer nivel de atención. ${ }^{18}$

Se calculó la ji cuadrada de Pearson para captar diferencias significativas respecto del uso del hospital para cualquier tipo de consulta, y sólo para consultas preventivas, entre beneficiarios agru- 
pados por patrones de uso de servicios y perfiles municipales de implementación del Seguro.

Este estudio fue aprobado por el Comité de Evaluación Ética del Instituto de la Salud Juan Lazarte. Se obtuvo el consentimiento informado de todos los sujetos participantes del estudio.

\section{Resultados}

Se realizaron 2413 entrevistas -de 296 a 309 por municipio. Las madres de los beneficiarios respondieron 98.3\% de dichas entrevistas. El nivel educativo de $80.8 \%$ de los respondientes fue igual o menor a siete años de estudio. Por otra parte, $19.2 \%$ de los niños de referencia eran menores de dos años (cuadro I).

El cuadro II se muestran los porcentajes de beneficiarios que obtuvieron algún tipo de atención médica durante el último año y que utilizaron el Seguro para alguna consulta, según el perfil municipal de implementación del Seguro. En la última columna se demuestra la existencia de asociaciones significativas entre la obtención de atención médica y el uso del Seguro con el perfil municipal de implementación del Seguro. Pese a la significancia estadística, el acceso a los servicios médicos fue elevado en todos los perfiles municipales y las diferencias en la obtención de atención fueron acotadas (promedio 97.2\% DE \pm 1.78 ). En contraste, las diferencias en el uso del Seguro fueron sustanciales (promedio 59.0\% DE \pm 23.66 ). Los principales motivos de no utilización del Seguro $(n=923)$ fueron: desconocimiento de ser beneficiario del Seguro $(44.2 \%$; $n=408)$ y preferencia por continuar atendiéndose con su médico de confianza $(41.6 \% ; \mathrm{n}=384)$.

El cuadro III muestra que $91.3 \%$ de los niños tuvieron acceso a consultas preventivas, mientras que menos de la mitad obtuvieron atención curativa. El grupo Seguro exclusivo, tuvo un acceso significativamente me-

\section{Cuadro II}

Porcentaje de encuestados Que obtuvieron atención MÉdICA (UTILIZANDO CUALQUIER FUENTE DE ATENCIÓN O Sólo UTILIZANDO LOS SERVICIOS DEL SEGURO), AL MENOS UNA VEZ DURANTE EL ÚLTIMO AÑO SEGÚN PERFILES municipales de implementación. Provincia de Buenos Aires, 2006.

\begin{tabular}{lcc}
$\begin{array}{l}\text { Perfiles municipales de implementación } \\
\text { del Seguro }\end{array}$ & $\begin{array}{c}\text { Obtuvo } \\
\text { atención }\end{array}$ & $\begin{array}{c}\text { Utilizó el Seguro } \\
\text { (seguro exclusivo o } \\
\text { seguro y otros) }\end{array}$ \\
Implementación integrada & $\begin{array}{l}98.0 \% \\
\mathrm{n}=59 \mid\end{array}$ & $\begin{array}{c}65.5 \% \\
\mathrm{n}=395\end{array}$ \\
\hline Implementación superpuesta & $96.4 \%$ & $71.9 \%$ \\
\hline Implementación paralela & $\mathrm{n}=582$ & $\mathrm{n}=434$ \\
\hline Implementación competitiva & $100 \%$ & $67.5 \%$ \\
& $\mathrm{n}=302$ & $\mathrm{n}=204$ \\
\hline Rechazo a la implementación & $96.9 \%$ & $57.5 \%$ \\
\hline Porcentaje & $\mathrm{n}=586$ & $\mathrm{n}=348$ \\
Valor absoluto & $95.3 \%$ & $14.0 \%$ \\
Desviación estándar & $\mathrm{n}=285$ & $\mathrm{n}=42$ \\
\hline Ji cuadrada de Pearson entre las cinco & $97.2 \%$ & $59.0 \%$ \\
categorías de municipios cuatro grados & $\mathrm{n}=2346$ & $\mathrm{n}=1423$ \\
de libertad & $\mathrm{D}=16.78$ & $\mathrm{DE} \pm 23.603$ \\
\hline
\end{tabular}

nor a consultas curativas en comparación con el grupo Seguro y otros. El grupo otros servicios tuvo un patrón de uso similar al del grupo Seguro exclusivo. El análisis del tipo de atención obtenida según las tres categorías de patrones de utilización se repitió para cada perfil municipal. La misma tendencia se verificó en los cinco casos con niveles de significancia estadística. Pese a esta tendencia general, se hallaron diferencias significativas en los patrones de uso y el acceso a los distintos tipos de atención entre los cinco casos $\left(\chi^{2}=210.882 ; p=0.000\right)$. Los beneficiarios de los casos de implementación integrada

Cuadro III

Comparación del tipo de ATENCIÓn ReCIBIDA SEgún PATRONES AÑo 2006

Proyecto “Análisis de las capacidades institucionales y eValuación del desempeño del SPS”. Provincia de Buenos aires, 2006

\begin{tabular}{|c|c|c|c|c|c|}
\hline Tipo de atención & Total & Seguro exclusivo & Seguro y otros & Otros servicios & Ji cuadrada de Pearson \\
\hline Sólo preventiva & $\begin{array}{c}51.7 \% \\
n=1212\end{array}$ & $\begin{array}{l}60.8 \% \\
n=603\end{array}$ & $\begin{array}{c}20.4 \% \\
n=88\end{array}$ & $\begin{array}{l}56.4 \% \\
n=521\end{array}$ & \multirow{4}{*}{$\begin{array}{l}\chi^{2}=321.9 \\
p=0.000\end{array}$} \\
\hline Sólo curativa & $\begin{array}{c}8.7 \% \\
n=204\end{array}$ & $\begin{array}{l}6.7 \% \\
n=66\end{array}$ & $\begin{array}{l}3.5 \% \\
n=15\end{array}$ & $\begin{array}{c}5.2 \% \\
n=123\end{array}$ & \\
\hline Preventiva y curativa & $\begin{array}{l}39.6 \% \\
n=930\end{array}$ & $\begin{array}{l}32.5 \% \\
n=322\end{array}$ & $\begin{array}{l}76.2 \% \\
n=329\end{array}$ & $\begin{array}{l}30.2 \% \\
n=279\end{array}$ & \\
\hline Total & $\begin{array}{c}100 \% \\
n=2346\end{array}$ & $\begin{array}{r}100 \% \\
n=991\end{array}$ & $\begin{array}{r}100 \% \\
n=432\end{array}$ & $\begin{array}{r}100 \% \\
n=923\end{array}$ & \\
\hline
\end{tabular}


y superpuesta tuvieron mayor acceso a una atención integral tanto en promedio como al interior del grupo de usuarios exclusivos y el grupo Seguro y otros.

Entre los usuarios del Seguro, $30.7 \%$ refirieron haber experimentado dificultades para ser atendidos, ya fuera debido a complicaciones para obtener el turno, o por los tiempos de espera. Las madres de los beneficiarios afirmaron que tanto los médicos del Seguro como aquellos de los servicios municipales promovían la realización de controles mediante estrategias como la asignación de días u horarios exclusivos para controles. Las entrevistadas afirmaron que resultaba mucho más difícil obtener turnos no programados para atender problemas de salud, que obtener turnos para controles.

En el cuadro IV se muestra el promedio anual de consultas preventivas y curativas según patrones de uso de servicios. Los beneficiarios del grupo Seguro exclusivo realizaron significativamente menos consultas preventivas ( 3.9 consultas anuales, $\mathrm{DE} \pm 3.0$ ) que los beneficiarios del grupo Seguro y otros (4.8 consultas, $\mathrm{DE} \pm 3.6, p=0.000$ ) aunque en ambos casos el número de consultas podría considerase alto. El promedio de consultas curativas también fue significativamente menor en el grupo Seguro exclusivo (1.5 consultas anuales, DE \pm 3.0 ), frente a los grupos Seguro y otros (3.4 consultas, $\mathrm{DE} \pm 4.0 p=0.000$ ) y otros servicios ( 1.9 consultas, $\mathrm{DE} \pm 3.6$, $p=0.016$ ). El grupo de otros servicios tendió a comportarse como el grupo del Seguro exclusivo.

Dicha comparación se repitió para cada perfil municipal y se verificó la misma tendencia respecto a la distribución de las consultas curativas y preventivas entre las tres categorías de beneficiarios por utilización de servicios. La excepción estuvo dada por el caso de implementación paralela, donde los grupos del Seguro exclusivo y otros servicios tuvieron una tasa de consulta similar y mayor a la del grupo Seguro y otros. Es im- portante destacar que, debido a su modalidad de implementación, en el caso de implementación paralela sólo 2.6\% de los beneficiarios utilizaron tanto los servicios del Seguro como otros servicios. En contraste, $17.3 \%$ de beneficiarios en el caso de implementación integrada, y $35.5 \%$ en el caso de implementación superpuesta, hicieron uso de ambas fuentes de atención. En el cuadro $\mathrm{V}$ se muestra el porcentaje de niños que recurrieron a los hospitales públicos para obtener atención médica preventiva o curativa $(26.7 \%)$ y, específicamente, para la obtención de atención preventiva (17.4\%). Los datos indican que los usuarios del Seguro recurrieron de manera significativamente menor a los hospitales para consultas generales y preventivas, que los beneficiarios que recibieron atención sin utilizar el Seguro.

El uso del hospital para consultas generales y preventivas entre usuarios y no usuarios del Seguro se probó en cada uno de los perfiles municipales de implementación del Seguro y se verificó la misma tendencia. Se hallaron diferencias significativas en todos los casos, salvo en el de implementación integrada. Se hallaron diferencias significativas en el uso de hospitales para la obtención de atención preventiva o curativa, o sólo preventiva, entre perfiles municipales y grupos de utilización de servicios. Es probable que las diferencias se expliquen más por la oferta local de servicios públicos de primer nivel (conformada por servicios del Seguro y la red de servicios municipal) y la cercanía de la población a los hospitales, y menos por las características del proceso de implementación local del Seguro.

Los entrevistados revelaron que solían utilizar los hospitales cuando experimentaban dificultades para obtener turnos en el primer nivel de atención, a fin de garantizar a sus hijos la atención médica necesaria por problemas de salud. Refirieron que, en ciertos casos, preferían recurrir directamente a los hospitales para

Cuadro IV

\section{Comparación del número de consultas preventivas y CuRAtivas según Patrones de utilización del SEguro utilizando el test de ANOVA one way. Proyecto “Análisis de las capacidades institucionales y eValuación del desempeño del SPS”. Provincia de Buenos Aires, 2006.}

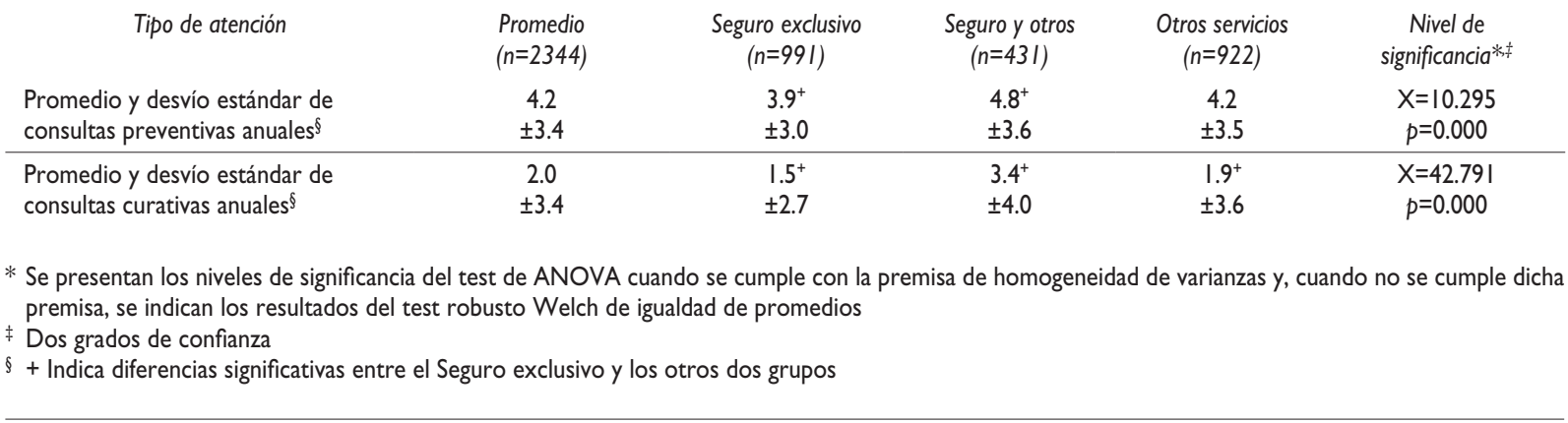




\section{Cuadro V \\ Uso de hospitales Públicos para la obtención de ATENCIÓn médicA entre usuarios del Seguro y de otros SERVICIOS por perfil municipal de implementación. Provincia de Buenos Aires, 2006. Proyecto “ANálisis de las capacidades institucionales y eVAluación del desempeño del SPS”}

Uso del hospital

\begin{tabular}{|c|c|c|c|c|}
\hline \multirow[b]{2}{*}{ Para todo tipo de consultas } & \multicolumn{4}{|c|}{$n=9 \angle 3$} \\
\hline & $26.7 \%$ & $19.9 \%$ & $36.6 \%$ & $\begin{aligned} \chi^{2} & =80.500 \\
p & =0.000\end{aligned}$ \\
\hline $\begin{array}{l}\text { Ji cuadrada de Pearson entre perfiles municipales de implementación cuatro } \\
\text { grados de libertad }\end{array}$ & $\begin{aligned} \chi^{2} & =103.946 \\
p & =0.000\end{aligned}$ & $\begin{aligned} \chi^{2} & =82.398 \\
p & =0.000\end{aligned}$ & $\begin{aligned} \chi^{2} & =42.443 \\
p & =0.000\end{aligned}$ & \\
\hline Sólo para consultas preventivas & $17.4 \%$ & $10.0 \%$ & $29.1 \%$ & $\begin{array}{c}\chi^{2}=128.376 \\
p=0.000\end{array}$ \\
\hline $\begin{array}{l}\text { Ji cuadrada de Pearson entre perfiles municipales de implementación cuatro } \\
\text { grados de libertad }\end{array}$ & $\begin{aligned} \chi^{2} & =56.886 \\
p & =0.000\end{aligned}$ & $\begin{aligned} \chi^{2} & =39.238 \\
p & =0.000\end{aligned}$ & $\begin{aligned} \chi^{2} & =24.963 \\
p & =0.000\end{aligned}$ & \\
\hline
\end{tabular}

obtener atención curativa, evitando el primer nivel de atención ya que sabían que, aunque en el hospital tendrían que esperar más tiempo, finalmente obtendrían la atención que necesitaban. Por último, refirieron que los profesionales del hospital solían citarlos para continuar allí los controles de salud y que cuando establecían un buen vínculo con los profesionales del hospital, acudían a ellos en forma espontánea para realizar los controles de salud de todos sus hijos.

\section{Discusión}

La evidencia indica que el Seguro logró mejorar las condiciones de acceso a los servicios de primer nivel de atención, dado que los perfiles municipales con una mayor proporción de cifras de usuarios del Seguro tuvieron porcentajes significativamente mayores de niños que recibieron atención. Sin embargo, inclusive en los municipios con bajos niveles de uso del Seguro el porcentaje de niños que obtuvieron atención fue elevado, tanto en términos absolutos como en comparación con los niveles de acceso referidos en otros estudios con población similar. ${ }^{18-20}$

El análisis del comportamiento de los perfiles municipales de implementación revela que el Seguro no logró garantizar en forma exclusiva una atención integral a la mayoría de sus beneficiarios. Quienes usaron el Seguro y otras fuentes, obtuvieron mejores resultados en cuanto a integralidad, a costa de una mayor fragmentación del proceso de atención. Por el contrario, quienes lograron una mayor continuidad de la atención tuvieron una menor tasa de consultas e integralidad de la atención. Distintos estudios sostienen que la asignación nominal de población a una determinada fuente de atención es un factor que mejora la continuidad de la atención, aunque supone desafíos en cuanto a las condiciones de accesibilidad. ${ }^{15,21}$ Esta premisa parece ser cierta en condiciones donde existen normas que limitan a la población el uso de servicios de salud distintos a los que están asignados.

Salvo en el caso de la implementación paralela, las restricciones para hacer uso de múltiples fuentes de atención son débiles, ineficientes o inexistentes. Como resultado, quienes tienen la capacidad suficiente para hacer uso de una mayor oferta de servicios logran un mejor acceso a una atención integral. ${ }^{18,22}$ Las modalidades de implementación que favorecieron la integralidad de la atención fueron aquellas donde el Seguro estuvo articulado con la red de servicios de salud municipales.

En consideración al hecho de que sólo la mitad de la población encuestada tuvo acceso a consultas curativas, mientras que más de $90 \%$ obtuvieron atención preventiva y que la tasa de consultas curativas fue de dos consultas anuales, se infiere que las barreras de acceso a la atención curativa constituyeron el principal limitante para el acceso a una atención integral. Un estudio realizado en Asturias, España, con niños de 0 a 6 años de edad, residentes en zonas urbanas, indica que en promedio se producen 4.8 episodios anuales de enfermedad por niño. ${ }^{23}$ En función de estos resultados y teniendo en cuenta que poblaciones en condiciones de alta vulnerabilidad socioeconómica, como las del presente estudio tienen todavía mayores posibilidades de enfermar, se puede afirmar que la tasa de consultas curativas es baja. La evidencia revelada a través de las entrevistas también respalda esta asunción y señala la existencia de un desbalance entre las condiciones de acceso a consultas preventivas y curativas. Este desbalance puede expli- 
carse por la tendencia a la programación y promoción de controles de salud y la consiguiente escasez de horas médicas para resolver y dar seguimiento a la atención de problemas de salud de la población. Esta situación puede explicarse como un efecto del modelo y normas de atención pediátricas del Ministerio y secretarías de salud de la Provincia de Buenos Aires, ${ }^{24}$ las exigencias de los programas sociales, que demandan el cumplimiento de los controles de niño sano para mantenerse bajo programa, y de los establecimientos escolares y deportivos, que imponen la presentación de certificados médicos como requisito de ingreso.

Durante las últimas décadas, organismos internacionales como la Organización Panamericana de la Salud y la Organización Mundial de Salud, el Banco Interamericano de Desarrollo y el Banco Mundial, entre otros, han ejercido una fuerte influencia en Latinoamérica para promover la atención preventiva mediante la introducción de programas sociales y de salud destinados a fortalecer la oferta de servicios preventivos y a incentivar la demanda de atención preventiva. ${ }^{25}$ Tanto los programas como la postulación de la prevención como bandera y prioridad de la atención primaria de la salud, han dejado una profunda impronta en la elaboración de normas asistenciales y el diseño de las políticas de salud en la región. ${ }^{25,26}$ Estos múltiples condicionamientos se traducen, en lo que respecta a la oferta de servicios, en una importante asignación de horas médicas para la prestación de controles de salud preventivos en un contexto donde la disponibilidad horaria semanal es relativamente baja, inclusive para cubrir los objetivos programáticos del control del lactante sano. ${ }^{24}$

Carr-Hill y colaboradores afirman que la experiencia en el uso de los servicios es un factor que moldea la percepción de la disponibilidad de estos últimos, y que dicha experiencia constituye un importante determinante de la demanda de servicios. ${ }^{27}$ Esta premisa aunada a los relatos de las entrevistadas sobre sus dificultades para acceder a la curativa en el primer nivel, proporciona una explicación sobre el motivo por el cual se produce una utilización tan elevada de los hospitales para la atención de problemas de salud banales. Es posible que las barreras de acceso al primer nivel para la obtención de atención curativa y la subsecuente utilización de los hospitales para consultas banales estén favoreciendo la generación de lazos de confianza, en virtud de lo cual es posible explicar tan altos porcentajes de uso de los hospitales para controles de salud preventivos. En este marco, el Seguro, mediante un aumento de la oferta de servicios de primer nivel y a través de la asignación nominal de pacientes a un proveedor desempeñó un rol fundamental en la disminución del uso de los hospitales para consultas banales y preventivas y, por lo tanto, fortaleció el primer nivel de atención como puerta de entrada y fuente habitual de atención.

En este artículo se pone de manifiesto que, en el marco de los sistemas de salud cuya prestación y gestión se encuentran descentralizadas en los municipios: a) la modalidad de implementación local de los programas provinciales o nacionales afecta su capacidad para generar mejoras en la calidad de la atención; b) la modalidad articulación de los servicios del programa y los servicios locales tiene implicaciones sobre los patrones de utilización de servicios, la integralidad de la atención y el nivel de fragmentación al interior del primer nivel; c) las mejoras en el acceso logradas a través de una ampliación de la oferta de servicios, no necesariamente traen aparejadas mejoras en la integralidad de la atención; d) un desbalance en el énfasis sobre la realización de controles de salud, puede generar barreras de acceso a la atención curativa y una pérdida de confianza de la población en el primer nivel de atención, y e) un mayor acceso a los servicios de primer nivel de atención disminuye el uso del hospital y fortalece el papel del primer nivel de atención.

\section{Agradecimientos}

Los autores de este artículo agradecen el apoyo brindado por la Organización Panamericana de la Salud (OPS) y el Centro Internacional de Investigaciones para el Desarrollo (IDRC), especialmente a Eduardo Levcovitz y Soledad Urrutia de la OPS, y a Roberto Bazzani y Adriana Bordabehere del IDRC. Asimismo, agradecen a Carlos Bloch, Director del Instituto de la Salud Juan Lazarte, su confianza y respaldo continuos y los aportes y la colaboración brindados por Adolfo Sánchez de León, Rafael Ventaffrida, Aurelia Furnari, Juan Herrman, Néstor Ponce, Nelva Cannelli y Gabriela Nestares, así como al resto de las personas que participaron en la planificación del estudio, la recolección y el procesamiento de datos y la discusión de la información.

Declaración de conflicto de intereses: Los autores declararon no tener conflicto de intereses.

\section{Referencias}

I. Programa Médicos Comunitarios. [sitio de internet]. Buenos Aires: Ministerio de Salud, [actualizado 2012 ene I2; consultado 2012 ene I2] Disponible en: http://www.msal.gov.ar/medicoscomunitarios/. 2. Ministerio de Salud y Deportes. Salud Familiar Comunitaria Intercultural. Documento Técnico - Estratégico. Versión didáctica. Publicación 17I. La Paz: Ministerio de Salud y Deportes, 2009. 3. Programas de agentes comunitários e Saúde da Familia: PACS/PSF. [sitio de internet]. San Pablo: Ministro de Estado da Saúde. Ministerio del Poder Popular de Planificación y Finanzas; 1997 [actualizado 2012 ene 
12; consultado 2012 ene 12] Disponible en: http://saudeprev.com.br/psf/ saopaulo/pdf-geral/Portaria\%20n\%BA\%20|886\%20Programas\%20de\%20 ACS-\%20PACS\%20\&PSF.htm.

4. Salud a su Casa [sitio de internet]. Bogotá: Secretaría de Salud. [actualizado 2012 ene 12; consultado 2012 ene I2] Disponible en: http:// www.saludcapital.gov.co/paginas/saludasuhogar.aspx.

5. Misión Barrio Adentro. [sitio de internet]. Caracas: Ministerio del Poder Popular de Planificación y Finanzas. [actualizado 2012 ene 12; consultado 2012 ene 12] Disponible en: http://fegs.gerenciasocial.org.ve/paginas/ RMISION_BARRIO_ADENTRO_I.html.

6. Organización Panamericana de la Salud / Organización Mundial de la Salud (OPS/OMS). Redes Integradas de Servicios de Salud: Conceptos, opciones de política y hoja de ruta para su implementación en las Américas. Washington (DC): Organización Panamericana de la Salud / Organización Mundial de la Salud, 2008.

7 Cetrángolo O, Devoto F. Organización de la salud en Argentina y equidad. Una reflexión sobre las reformas de los años noventa e impacto de la crisis actual. Toronto: Comisión Económica para América Latina, 2002. 8. Ministerio de Salud de la Provincia de Buenos Aires (MSPBA). Manual del Médico de Cabecera del Seguro Público de Salud de la Provincia de Buenos Aires. La Plata: Seguro Público de Salud de la Provincia de Buenos Aires, 2003.

9. Evans P. El Estado como problema y como solución. Desarrollo Econ 1996; 35(I40):529-562.

10. Báscolo E, Yavich N. Governance and effectiveness of the Buenos Aires Public Health Insurance implementation process. J Ambulatory Care Manage 2009; 32(2):91-102.

II. Báscolo E, Yavich N. Gobernanza y gobernabilidad del Seguro Público de Salud de la Provincia de Buenos Aires. En: Hernández A, Rico C, eds. Protección social en salud en América Latina y el Caribe: investigación y políticas. Bogotá: Editorial Pontificia Universidad Javeriana, 20 I I:159- 885 12. Censo Nacional de Población, Hogares y Vivienda 2010. Cuadro PI-P. Provincia de Buenos Aires e interior de la Provincia. Población total y variación intercensal absoluta y relativa por partido. Años 200 I20I0. Instituto Nacional de Estadísticas y Censos. [Consultado enero I2, 2012] Disponible en http://www.censo 2012.indec.gov.ar/resultados definitivos. asp.

13. Sánchez de León A. La función de compra de servicios de salud en el marco del Seguro Público de Salud de la Provincia de Buenos Aires. En: Báscolo E, comp. Seguro Público de Salud. Aportes para un debate abierto. Rosario: Instituto de la Salud Juan Lazarte, 2004: I0 I- I I4.

14. Mendoza-Sassi R, Béria JU. Utilización de los servicios de salud: una revisión sistemática sobre los factores relacionados. Cad Saude Pública 200I; I7(4):819-832.

15. Haggerty J, Burge F, Levesque JF, Gass D, Pineault R, Beaulieu MD, et al. Operational definitions of attributes of primary health care: consensus among Canadian experts. Ann Fam Med 2007; 5:336-344.
16. Tellez TA, Aguayo TE. Resultados de un sistema de atención privado basado en un modelo de salud familiar en Chile. Rev MedChil 2008; 136(7): 873-879.

17. Starfield B. El cupo y la longitudinalidad. En: Atención primaria. Equilibrio entre necesidades de salud, servicios y tecnología. Barcelona: Masson, 200I:|53-8|.

18. Báscolo E, Yavich N, Urquía M. Capacidades locales de gestión como factor predictivo de la utilización y accesibilidad a los servicios de primer nivel para la obtención de cuidados prenatales. Rev Gerenc Polit Salud 2007; 6(I2): III2-125.

19. Departamento de Economía y Gestión en Salud (DEGES). Resultados de la Encuesta de Accesibilidad en el Municipio de Rosario. Boletín Gobernanza y Atención Primaria de la Salud [serie en internet] 2008 [consultado 2010 octubre I5]; 5:I-2. Disponible en: http://www.aps-gza. com.ar/boletin/Boletin-Gobernanza-Salud-5.pdf.

20. Departamento de Economía y Gestión en Salud (DEGES). Resultados de la Encuesta de Accesibilidad en el Municipio de General San Martín. Boletín Gobernanza y Atención Primaria de la Salud [serie en internet] 2009 [consultado 2010 octubre 15]; I4:I-2. Disponible en: http://www. aps-gza.com.ar/boletin/Boletin-Gobernanza-Salud- I4.pdf.

2I. Haggerty JL, Pineault R, Beaulieu MD, Brunelle Y, Gauthier J, Goulet $F$, et al. Practice features associated with patient-reported accessibility, continuity, and coordination of primary health care. Ann Fam Med 2008; 6:116-123.

22. Lewis M, Eskeland G, Traa-Valerezo X. Primary health care in practice: is it effective? Health Policy 2004; 70(3):303-325. 23. Oterino de la Fuente D, Martín-Díaz R, Díaz-Megido LA, Alvargonzález-Terrero $B$. Variaciones en el consumo de recursos de atención primaria por niños menores de 7 años en los medios rural y urbano. Estudio de cohortes. Aten Primaria 2003; 3 I (8):480-485. 24. Área de Programación. PROAPS - REMEDIAR. Control del lactante sano: un análisis desde ópticas diferentes. Boletín PROAPS - REMEDIAR [serie en internet] 2005 [consultado 2010 noviembre 15]; 3(19):5-8. Disponible en: http://www.femeba.org.ar/fundacion/quienessomos/ Novedades/boletinremediar 19.pdf.

25. Acuña C. Incentivos a la demanda como mecanismos de extensión de la protección social en salud [monografía en internet]. Washington: Organización Panamericana de la Salud / Organización Mundial de la Salud. Área de Fortalecimiento de los Sistemas de Salud, 2008. [consultado 2010 diciembre]. Disponible en: http://new.paho.org/hss/ index.php?gid=10538\&option=com_docman\&task=doc_download. 26. Organización Mundial de la Salud (OMS). Declaración de Alma-Ata. Conferencia Internacional sobre Atención Primaria de Salud; septiembre 6-12. Alma-Ata: Organización Mundial de la Salud (OMS), 1978. 27. Carr-Hill RA, Hardman G, Martin S, Peacock S, Sheldon TA, Smith P. A formula for distributing NHS revenues based on small area use of hospital beds. York: Centre for Health Economics, University of York, 1994. 